\title{
A proportional hazards regression model for the subdistribution with right-censored and left-truncated competing risks data
}

\author{
Xu Zhanga, ${ }^{a}, \dagger$, Mei-Jie Zhang ${ }^{\mathrm{b}}$, and Jason Fine ${ }^{\mathrm{c}}$ \\ aDepartment of Mathematics and Statistics, Georgia State University, Atlanta, GA 30303, U.S.A. \\ bDivision of Biostatistics, Medical College of Wisconsin, Milwaukee, WI 53226, U.S.A. \\ 'Department of Biostatistics, University of North Carolina at Chapel Hill, Chapel Hill, NC 27599, \\ U.S.A.
}

\begin{abstract}
With competing risks failure time data, one often needs to assess the covariate effects on the cumulative incidence probabilities. Fine and Gray proposed a proportional hazards regression model to directly model the subdistribution of a competing risk. They developed the estimating procedure for right-censored competing risks data, based on the inverse probability of censoring weighting. Right-censored and left-truncated competing risks data sometimes occur in biomedical researches. In this paper, we study the proportional hazards regression model for the subdistribution of a competing risk with right-censored and left-truncated data. We adopt a new weighting technique to estimate the parameters in this model. We have derived the large sample properties of the proposed estimators. To illustrate the application of the new method, we analyze the failure time data for children with acute leukemia. In this example, the failure times for children who had bone marrow transplants were left truncated.
\end{abstract}

\section{Keywords}

competing risks; cumulative incidence function; proportional hazards model; subdistribution

\section{Introduction}

For medical studies involving competing risks, clinicians often wish to estimate and model the cumulative incidence probability of a specific cause of failure. In a published study [1] researchers compared the effectiveness of chemotherapy versus bone marrow transplantation (BMT) on the leukemia-free survival (LFS) for children with acute lymphoblastic leukemia (ALL) in second complete remission. The transplant cohort consisted of data from the International Bone Marrow Transplant Registry (IBMTR). Only patients receiving transplants were included in the IBMTR cohort, thus, the time to failure was left truncated by the transplant time. Leukemia patients were subject to competing risks of treatment failures, cancer relapse and treatment-related mortality (TRM), which is defined as death in complete remission. To understand the effect of prognostic factors on composite treatment failure, it is necessary to disentangle their separate effects on the cumulative incidences of these competing risks. Such analyses must deal appropriately with the left truncation for the BMT individuals.

\footnotetext{
Copyright (@) 2011 John Wiley \& Sons, Ltd.

${ }^{\star}$ Correspondence to: Department of Mathematics and Statistics, Georgia State University, Atlanta, GA 30303, U.S.A. .

†matxxz@langate.gsu.edu.
} 
Traditionally, the standard approach for analyzing competing risks data has been to estimate and model the cause-specific hazards for all causes. Let $\lambda_{k}(t ; z)$ be the hazard of the $k$ th cause, conditional on the covariates $z$. Assuming $k=1,2$, the cumulative incidence function of cause 1 given $z$ is defined as

$$
F_{1}(t ; z)=P(T \leq t, \varepsilon=1 \mid z)=\int_{0}^{t} \exp \left[-\int_{0}^{s^{-}}\left\{\lambda_{1}(u ; z)+\lambda_{2}(u ; z)\right\} \mathrm{d} u\right] \lambda_{1}(s ; z) \mathrm{d} s,
$$

where $T$ is the failure time and $\varepsilon$ indicates the cause of failure. For the right-censored competing risks data, $F_{1}(t ; z)$ can be estimated by a plug-in estimator. Here, $\lambda_{k}(t ; z)$ must be modeled. Cheng et al. [2] considered the Cox model for both causes, Shen and Cheng [3] studied a special additive risk model, and recently, Scheike and Zhang [4, 5] proposed and studied a flexible Cox-Aalen model allowing some covariates to have time-varying effects. For left-truncated and right-censored competing risks data, the standard approaches to dealing with cause-specific hazard functions can be generalized to the left-truncated versions by adjusting the risk set [6].

For the standard approach, the covariate effect is assessed on each cause-specific hazard, creating a complex nonlinear modeling relationship for the cumulative incidence function. Fine and Gray [7] developed a method to directly model the cumulative incidence function by modeling a subdistribution hazard function, $\lambda_{1}^{*}(t ; z)=-\mathrm{d} \log \left\{1-F_{1}(t ; z)\right\} / \mathrm{d} t$. They proposed a proportional subdistribution hazards model,

$$
\lambda_{1}^{*}(t ; z)=\lambda_{10}^{*}(t) \exp \left\{\beta_{0}^{\mathrm{T}} z\right\} .
$$

The cumulative incidence function can be directly modeled as

$$
F_{1}(t ; z)=1-\exp \left\{-\exp \left(\beta_{0}^{\mathrm{T}} z\right) \int_{0}^{t} \lambda_{10}^{*}(s) \mathrm{d} s\right\}
$$

Sun et al. [8] and Scheike et al. [9] considered some alternative models for the subdistribution hazard. For the right-censored competing risks data, Fine and Gray proposed using an inverse probability of censoring weighting (IPCW) technique to estimate $\boldsymbol{\beta}_{0}$ and the cumulative baseline subdistribution hazard function $\Lambda_{10}^{*}(t)=\int_{0}^{t} \lambda_{10}^{*}(s) d s$, and derived large sample properties of proposed estimators.

It is unknown how to fit Fine and Gray's semiparametric subdistribution hazard model to left-truncated and right-censored competing risks data. The main focus of this paper is to find an appropriate weight for Fine and Gray's method. Directly adopting Fine and Gray's approach, one may consider using the left-truncated version Kaplan-Meier estimator of the censoring distribution for the weight. However, it can be easily seen that for an uncensored but truncated sample, the censoring probability weight equals to a constant 1 , which leads to an 'equal weight' estimating procedure. We showed that, with no covariates, the estimates of a subdistribution hazard with a constant weight is obviously biased when the failure times are left truncated [10]. This indicates that IPCW is not an appropriate weight for lefttruncated data.

In this paper, we derived a reciprocal of the truncation-censoring probability weight for a right-censored and left-truncated sample. We proposed two weights. The first weight estimator can be explained by a mass distribution algorithm [10], which leads to a standard left-truncated version of Aalen-Johansen's estimator for the cumulative incidence function 
for the no covariate case. The second weight was derived from the conditional censoring distribution after delayed entry time. We have derived large sample properties for the proposed estimators. The performances of these two weights were studied through simulation. The estimators based on the proposed weights are shown to be asymptotically unbiased.

The outline of the remainder of the paper is as follows. In Section 2, we describe the data structure. In Section 3, we develop the inverse weighted estimation for the proportional subdistribution hazards model. Simulation studies are given in Section 4. In Section 5, we analyze a real data set, which was originally studied by Barrett et al. [1], to give an application of the model of interest. Concluding remarks are given in Section 6.

\section{Left-truncated competing risks data}

Suppose that there are two competing risks. Let $T_{i}$ be the failure time and let $L_{i}$ and $C_{i}$ be the left truncation time and the right censoring time, respectively. $\varepsilon_{i} \in\{1,2\}$ indicates the cause of failure. For left-truncated and right-censored data, $X_{i}=\min \left(T_{i}, C_{i}\right)$ and $\Delta_{i}=I\left\{T_{i} \leq\right.$ $\left.C_{i}\right\}$ are observed only if $L_{i} \leq X_{i}$, where $I\{\cdot\}$ is the indicator function. Let $Z_{i}$ be the associated covariates. We assume that, given covariates $\boldsymbol{Z}_{i}, T_{i}$ is independent from $\left(L_{i}, C_{i}\right)$. The observed data $\left\{L_{i}, X_{i}, \Delta_{i}, \Delta_{i} \varepsilon_{i}, Z_{i}\right\}$ are independent and identically distributed for $i=1$, $\ldots, n$. Let $G_{L}$ be the distribution function of $L$.

For left-truncated and right-censored competing risks data, we define the underlying counting processes $N_{i}^{L, 1}(t)=I\left\{L_{i} \leq T_{i} \leq t, \varepsilon_{i}=1\right\}$ and a modified risk indicator $Y_{i}^{L, 1}(t)=I\left\{\left(L_{i} \leq t \leq T_{i}\right) \cap\left\{\left(L_{i} \leq T_{i} \leq t\right), \varepsilon_{i}=2\right\}\right\}$. Note that $N_{i}^{L, 1}(t)$ and $Y_{i}^{L, 1}(t)$ are not observable for all time $t$. Let $r_{i}(t)=I\left\{L_{i} \unlhd\left(T_{i} \wedge t\right) \leq C_{i}\right\}$, where $x \wedge y=\min (x, y)$, then observed counting process $N_{i}^{1}(t)=r_{i}(t) N_{i}^{L, 1}(t)=I\left\{L_{i} \leq X \leq t, \Delta_{i} \varepsilon_{i}=1\right\}$ and observed modified risk indicator $\left.Y_{i}^{1}(t)=r_{i}(t) Y_{i}^{L, 1}(t)=I\left\{L_{i} \leq t \leq X_{i}\right) \cup\left(L_{i} \leq X_{i} \leq t, \Delta_{i} \varepsilon_{i}=2\right)\right\}$ are computable for all time $t$.

\section{Inverse weight for right-censored and left-truncated competing risks data}

Estimation of the regression parameters in (1) with complete data follows the definition of a subdistribution hazard. Fine and Gray proposed maximizing the following partial likelihood:

$$
\prod_{i=1}^{n}\left[\frac{\lambda_{10}\left(T_{i}\right) \exp \left(\beta^{\mathrm{T}} \boldsymbol{Z}_{i}\right)}{\sum_{j \in R_{i}} \lambda_{10}\left(T_{i}\right) \exp \left(\beta^{\mathrm{T}} \boldsymbol{Z}_{j}\right)}\right]^{I\left(\varepsilon_{i}=1\right)},
$$

where $R_{i}$ is the risk set at time $T_{i}$ and is specially defined to include the alive subjects and the subjects failing from the other cause prior to $T_{i}$. For the right-censored competing risks data, Fine and Gray proposed using an inverse probability censoring weight and obtaining the MLE by solving the following weighted score estimating equation:

$$
\sum_{i=1}^{n} \int_{0}^{\infty}\left[\boldsymbol{Z}_{i}(t)-\frac{\sum_{j} \kappa_{j}(t) Y_{j}^{1}(t) \boldsymbol{Z}_{j} \exp \left(\beta^{\mathrm{T}} \boldsymbol{Z}_{j}\right)}{\sum_{j} \kappa_{j}(t) Y_{j}^{1}(t) \exp \left(\beta^{\mathrm{T}} \boldsymbol{Z}_{j}\right)}\right] \kappa_{i}(t) \mathrm{d} N_{i}^{1}(t)=0,
$$

where $Y_{i}^{L, 1}(t), N_{i}^{L, 1}(t), r_{i}(t)$ agree with the definitions in Section 2 with $L_{i}=0$, weight $\kappa_{i}(t)=r_{i}(t) \widehat{G}_{C}(t) / \widehat{G}_{C}\left(X_{i} \wedge t\right)$, and $\widehat{G}_{C}(t)$ is the Kaplan-Meier estimate of $P(C>t)$. The IPCW 
technique has been routinely adopted for inferences about the cumulative incidence function with right-censored competing risks data $[8,9]$.

We propose the new weights to adjust for censoring and truncation and employ a weighted score estimation equation similar to equation (2) with proposed weights. Let $G_{L, C}(t \mid Z)=$ $P(L \leq t \leq C \mid L \leq X, \boldsymbol{Z})$. The proposed weights can be explained by $E\left\{r_{i}(t) / G_{L, C}\left(X_{i} \wedge t \mid Z_{i}\right) \mid T_{i}\right.$, $\left.L_{i} \leq X_{i}, \varepsilon_{i}, Z_{i}\right\}=1$. In Sections 3.1 and 3.2, we introduce two inverse weights under the assumption that $T_{i}$ is independent of $L_{i}$ and $C_{i}$ given $Z_{i}$. The large sample inferences are given in Section 3.3.

\subsection{Weight 1}

In Appendix A, we show that $G_{L, C}\left(X_{i} \wedge t \mid Z_{i}\right)=b\left(X_{i} \wedge t \mid Z_{i}\right) / S\left(X_{i}^{-} \wedge t^{-} \mid Z_{i}\right)$, where $b\left(t \mid Z_{i}\right)=$ $P\left(L_{i} \leq t \leq X_{i} \mid L_{i} \leq X_{i}, Z_{i}\right)$ and $S\left(t \mid Z_{i}\right)=P\left(T_{i}>t \mid Z_{i}\right)$. It follows that $G_{L, C}\left(X_{i} \wedge t \mid Z_{i}\right)$ can be estimated

$$
\widehat{G}_{L, C}\left(X_{i} \wedge t \mid Z_{i}\right)=\frac{\widehat{b}\left(X_{i} \wedge t \mid Z_{i}\right)}{\widehat{S}\left(X_{i}^{-} \wedge t^{-} \mid Z_{i}\right)},
$$

where $\widehat{b}\left(t \mid Z_{i}\right)$ and $\widehat{S}\left(t \mid Z_{i}\right)$ are the predicted estimators based on regression models. Here, we derived a time-dependent weight

$$
\widehat{w}_{i}\left(t \mid Z_{i}\right)=\frac{r_{i}(t)}{\widehat{G}_{L, C}\left(X_{i} \wedge t \mid Z_{i}\right)}=\frac{r_{i}(t) \widehat{S}\left(X_{i}^{-} \wedge t^{-} \mid Z_{i}\right)}{\widehat{b}\left(X_{i} \wedge t \mid Z_{i}\right)} .
$$

Following Fine and Gray's [7] approach, we consider a modified weight by multiplying a term $\widehat{b}(t) / \widehat{S}\left(t^{-}\right)$to $\widehat{w}_{i}(t)$

$$
\widehat{w}_{i}^{(1)}\left(t \mid Z_{i}\right)=\frac{r_{i}(t) \widehat{S}\left(X_{i}^{-} \wedge t^{-} \mid Z_{i}\right) \widehat{b}(t)}{\widehat{b}\left(X_{i} \wedge t \mid Z_{i}\right) \widehat{S}\left(t^{-}\right)},
$$

where $\widehat{b}(t)=n^{-1} \sum_{i} I\left\{L_{i} \leq t \leq X_{i}\right\}, \widehat{S}(t)$ is the left-truncated version of Kaplan-Meier estimator for the overall survival.

\subsection{Weight 2}

The left truncation time can be considered as delayed entry time since we observe only subjects from truncation time. Let $T=L+\widetilde{T}$ and $C=L+\widetilde{C}$. In theory, $\widetilde{T}$ and $\widetilde{C}$ may take negative values, although data are observed only if $\widetilde{X}=(\widetilde{T} \wedge \widetilde{C})=(T \wedge C)-L \geq 0$, i.e. only if $\widetilde{T} \geq 0$ and $\widetilde{C} \geq 0$. In Appendix A, we show that

$$
G_{L, C}(X \wedge t \mid Z)=G_{\widetilde{c} \tilde{x} \geq 0}\{(X \wedge t)-L \mid Z\} \times K(X \wedge t \mid Z),
$$

where $G_{\widetilde{c} \widetilde{X} \geq 0}(t \mid Z)=P(\widetilde{C} \geq 0 \mid \widetilde{X} \geq 0, t \geq 0, Z)$ and $K(t \mid Z)=P(L \leq \mid L \leq X, \boldsymbol{Z})$. 
Note that one can estimate $G_{\widetilde{c} \tilde{\mathrm{X}} \geq 0}(t \mid \boldsymbol{Z})$ using the right-censored data $\left\{X_{i}-L_{i}, 1-\Delta_{i}, \boldsymbol{Z}_{i} ; i=1\right.$, $\ldots, n\}$ and denoted as $G_{\tilde{C} \mid \tilde{x} \geq 0}(t \mid Z)$ and $K(t \mid Z)$ can be estimated based on observed data $\left\{L_{i}\right.$, $\left.Z_{i}, i=1, \ldots, n\right\}$ and denoted as $\widehat{K}(t \mid Z)$. This leads to an alternative weight

$$
\widehat{w}_{i}^{(2)}\left(t \mid Z_{i}\right)=\frac{r_{i}(t)}{\widehat{G}_{\tilde{C} \tilde{X} \geq 0}\left\{\left(X_{i} \wedge t\right)-L_{i} \mid Z_{i}\right\} \times \widehat{K}\left(X_{i} \wedge t \mid Z_{i}\right)} .
$$

The proposed weights are unknown in practice. It can be estimated nonparametrically if the weight function is independent of the covariates, i.e. $G_{L, C}(t \mid \mathbf{Z})=P(L \leq t \leq C \mid L \leq X, \boldsymbol{Z})=$ $P(L \leq \leq C \mid L \leq X)$. When the weight depends on the covariates, it needs to be estimated by a predicted value for each individual based on regression models, such as Aalen's additive regression model. If there are few discrete covariates, a stratified nonparametric weight estimator can be used. For simplicity, we present nonparametric weight estimators

$$
\begin{gathered}
\widehat{w}_{i}^{(1)}(t)=\frac{r_{i}(t) \widehat{S}\left(X_{i}^{-} \wedge t^{-}\right) \widehat{b}(t)}{\widehat{b}\left(X_{i} \wedge t\right) \widehat{S}\left(t^{-}\right)}, \\
\widehat{w}_{i}^{(2)}(t)=\frac{r_{i}(t)}{\widehat{G}_{\widetilde{c} \tilde{X} \geq 0}\left\{\left(X_{i} \wedge t\right)-L_{i}\right\} \times \widehat{K}\left(X_{i} \wedge t\right)},
\end{gathered}
$$

where $G_{\widetilde{C} \tilde{X} \geq 0}(t)$ is the Kaplan-Meier estimator using the right-censored sample $\left\{X_{i}-L_{i}, 1-\right.$ $\left.\Delta_{i}, i=1, \ldots, n\right\}$, and $\widehat{K}(t)=n^{-1} \sum_{i} I\left\{L_{i} \leq t\right\}$.

\section{Remarks-}

1. For the left-truncated and right-censored competing risks data, when there is no covariate presented, the cumulative incidence function $F_{1}(t)$ is commonly estimated by an Aalen-Johansen estimator

$$
\widehat{F}_{1}^{A J}(t)=\int_{0}^{t} \widehat{S}\left(u^{-}\right) \mathrm{d} \widehat{\Lambda}_{1}(u),
$$

where $\widehat{\Lambda}_{1}(u)$ is left-truncated version Nelson-Aalen estimator for cause 1 specific hazard. Adopting Efron's [11] redistribution-to-right technique to the left-truncated and right-censored competing risks data, we showed that $F_{1}(t)$ can be estimated by a product-limit estimator of the subdistribution hazard using weight 1 given in (5)

$$
\widehat{F}_{1}^{P L}(t)=1-\prod_{u \leq t}\left\{1-\frac{\sum_{i=1}^{n} \widehat{w}_{i}^{(1)}(u) d N_{i}^{L, 1}(u)}{\sum_{j=1}^{n} \widehat{w}_{j}^{(1)}(u) Y_{j}^{L, 1}(u)}\right\},
$$

and we showed that $\widehat{F}_{1}^{A J}(t)=\widehat{F}_{1}^{P L}(t)$ (see [10] for detail). However, the productlimit estimator based on the alternative weight, $\widehat{w}_{i}^{(2)}(t)$, is not identical to the standard Aalen-Johansen's nonparametric estimator of $\widehat{F}_{1}^{A J}(t)$. 
2. We noted that model mis-specification is a critical issue for the covariate adjusted weight. Some exploratory study suggests the utilization of Aalen's model for the covariate adjusted weight.

3. Our aim of converting $\widehat{w}_{i}\left(t \mid Z_{i}\right)$ to $\widehat{w}_{i}^{(1)}\left(t \mid Z_{i}\right)$ is to reduce the high variability contained in the original weight. The final nonparametric weight, $\widehat{w}_{i}^{(1)}(t)$, agrees with the stabilized form of the IPCW weights adopted in the same model for rightcensored data [7]. The initial application of IPCW is characterized by the inverse of a censoring probability [12]. In dealing with dependent censoring, Robins and Finkelstein [13] aimed to discover the effects of covariate processes, $\boldsymbol{V}(t)$, and suggested a different IPCW weight $\widehat{G}_{C}(t) / \widehat{G}_{C}(t ; \boldsymbol{V}(t))$. For this weight, $\widehat{G}_{C}(t)$ is the Kaplan-Meier estimate and $\widehat{G}_{C}(t ; \boldsymbol{V}(t))$ is the adjusted survival estimate given the covariate processes up to time $t$.They stated that the weight $1 / \widehat{G}_{C}(t ; \boldsymbol{V}(t))$ can be alternatively used, but the first IPCW weight has the advantage of efficiency. The term 'stabilized weight' appeared in the work on the causal inference of treatments [14] (see Section 6.1), where reduction in variability was mentioned as the motivation for adopting weight in the stabilized form.

4. Similarly, the stabilized form for weight 2 can be considered

$$
\frac{r_{i}(t) \widehat{G}_{\widetilde{C} \widetilde{X} \geq 0}\left\{t-L_{i}\right\} \times \widehat{K}(t)}{\widehat{G}_{\widetilde{C} \widetilde{X} \geq 0}\left\{\left(X_{i} \wedge t\right)-L_{i}\right\} \times \widehat{K}\left(X_{i} \wedge t\right)} .
$$

A limited simulation indicates that using this modified weight, the variability among the regression coefficient estimates has been greatly reduced. However, the bias becomes much greater (see Table III). More investigation is needed to find out the stabilized form for weight 2 .

\subsection{Large sample inferences}

The proposed weights can be utilized to estimate the parameters in model (1) by solving a weighted score estimating equation (2). For simplicity, we present large sample results based on nonparametric estimated weights. Similar asymptotic results can be derived for covariate adjusted weights which depend on the regression models used in the weight estimation. For weight $\widehat{w}_{i}^{(k)}(t), k=1,2, \boldsymbol{\beta}$ can be estimated by solving $\boldsymbol{U}^{(k)}\left(\widehat{\beta}^{(k)}\right)=0$, where

$$
\boldsymbol{U}^{(k)}(\beta)=\sum_{i=1}^{n} \int_{0}^{\infty}\left\{\boldsymbol{Z}_{i}-\frac{\sum_{j=1}^{n} \widehat{w}_{j}^{(k)}(t) Y_{j}^{L, 1}(t) \boldsymbol{Z}_{j} \exp \left(\boldsymbol{Z}_{j}^{\mathrm{T}} \beta\right)}{\sum_{j=1}^{n} \widehat{w}_{j}^{(k)}(t) Y_{j}^{L, 1}(t) \exp \left(\boldsymbol{Z}_{j}^{\mathrm{T}} \beta\right)}\right\} \times \widehat{w}_{i}^{(k)}(t) \mathrm{d} N_{i}^{L, 1}(t) .
$$

The cumulative baseline subdistribution hazards can be estimated by

$$
\widehat{\Lambda}_{10}^{*(k)}(t)=\sum_{i=1}^{n} \int_{0}^{t} \frac{\widehat{w}_{i}^{(k)}(u) d N_{i}^{L, 1}(u)}{\sum_{j} \widehat{w}_{j}^{(k)}(u) Y_{j}^{L, 1}(u) \exp \left(Z_{j}^{\mathrm{T}} \widehat{\beta}^{(k)}\right)} .
$$

Using the basic empirical process theory as in Fine and Gray [7], we show briefly in Appendix B that, under the standard regular conditions [15] and assumption that $T_{i}$ is 
independent of $\left(L_{i}, C_{i}\right)$ given $\boldsymbol{Z}_{i}, \widehat{\beta}^{(k)}$ is a consistent estimator. $n^{1 / 2}\left(\widehat{\beta}^{k)}-\beta_{0}\right)$ converges in distribution to a zero-mean Gaussian random vector, and its covariance matrix can be consistently estimated by

$$
\widehat{\Sigma}_{\beta}^{(k)}=\left\{n^{-1} \boldsymbol{I}^{(k)}\left(\widehat{\beta}^{(k)}\right)\right\}^{-1} \times\left\{n^{-1} \sum_{i}\left(\widehat{\boldsymbol{W}}_{\beta, i}^{(k)}\right)^{\otimes 2}\right\} \times\left\{n^{-1} \boldsymbol{I}^{(k)}\left(\widehat{\beta}^{(k)}\right)\right\}^{-1},
$$

where explicit expressions for $\boldsymbol{I}^{(k)}(\boldsymbol{\beta})$ and $\widehat{\boldsymbol{W}}_{\beta, i}^{(k)}$ are given in Appendix B.

Furthermore, we can show that $n^{1 / 2}\left\{\widehat{\Lambda}_{10}^{*(k)}(t)-\Lambda_{10}^{*}(t)\right\}$ converges weakly to a zero-mean Gaussian process, and the variance function can be consistently estimated by

$$
\widehat{\Sigma}_{\Lambda_{10}^{*}}^{(k)}(t)=n^{-1} \sum_{i=1}^{n}\left\{\widehat{W}_{\Lambda_{10}^{*}, i}^{(k)}(t)\right\}^{2},
$$

where explicit expressions for $\widehat{W}_{\Lambda_{10}^{*}, i}^{(k)}(t)$ are given in Appendix C.

The predicted cumulative incidence curve for a given set of covariate values is an important summary curve to show the treatment efficacy for a particular cause of failure over time. It can be predicted by a plug-in estimator

$$
\widehat{F}_{1}^{(k)}(t ; z)=1-\exp \left\{-\exp \left(z^{\mathrm{T}} \widehat{\beta}^{(k)}\right) \int_{0}^{t} \mathrm{~d} \widehat{\Lambda}_{10}^{*(k)}(u)\right\} .
$$

Using the functional delta method, we show that $n^{1 / 2}\left\{\widehat{F}_{1}^{(k)}(t ; z)-F_{1}(t ; z)\right\}$ converges weakly to a zero-mean Gaussian process, and the variance function can be estimated by

$$
\widehat{\Sigma}_{F_{1}}^{(k)}(t)=n^{-1}\left\{1-\widehat{F}_{1}^{(k)}(t ; z)\right\}^{2} \sum_{i=1}^{n}\left\{\widehat{W}_{F_{1}, i}^{(k)}(t ; z)\right\}^{2}
$$

where explicit expressions for $\widehat{W}_{F_{1}, i}^{(k)}(t ; z)$ are given in Appendix D.

\subsection{The censoring/truncation time is associated with some covariates}

In this subsection, we consider the methods to estimate the covariates adjusted weight. When the censoring/truncation time depends on some discrete covariates, one can utilize the stratified nonparametric weight as given below. The data set can be summarized as $\left\{L_{r i}, X_{r i}\right.$, $\left.\Delta_{r i}, \Delta_{r i} \varepsilon_{r i}, \boldsymbol{Z}_{r i}\right\}$, for $r=1, \cdots, R$, and $i=1, \cdots, n_{r}$, where $R$ is the number of strata. Let $\widehat{S}_{r}(t)$ and $\widehat{b}_{r}(t)$ be the relevant estimators for the $t$ th strata. The stratified version of weight 1 has the form,

$$
\widetilde{w}_{r i}(t)=\frac{r_{r i}(t) \widehat{S}_{r}\left(X_{r i}^{-} \wedge t^{-}\right) \widehat{b}(t)}{\widehat{b}_{r}\left(X_{r i} \wedge t\right) \widehat{S}\left(t^{-}\right)} .
$$


As shown in Appendices B-D, variances of the regression parameter estimators consist of two parts. The first part corresponds to a model where the weight function is known. Scheike et al. [9] showed that the results based only on the first part lead to slightly conservative, but acceptable variance estimators. For simplicity, we present variance estimation based only on the major (first) part for stratified weight (see Appendix E for details). In our simulation study, we show that this simplified variance estimation approach leads to acceptable results.

When the censoring/truncation time is associated with a large number of covariates, one should consider constructing appropriate regression models and find the covariate adjusted weight. The covariate adjusted version of weight 1 requires estimation of $P(T>\mid z), P(L>t \mid$ $L \leq X, z)$ and $P(X>t \mid L \leq X, z)$. We consider Aalen's model as the underlying regression models allowing time-varying effects. Let $\lambda_{T}(t ; z), \lambda_{L / L \leq X}(t ; z), \lambda_{X \mid L \leq X}(t ; z)$ be the hazard rate functions of the relevant random variables. We use the estimators $\widehat{S}_{T}(t ; z)=\exp \left\{-\int_{0}^{t} \widehat{\lambda}_{T}(t ; z)\right\}$, $\widehat{S}_{L L \leq X ; z}=\exp \left\{-\int_{0}^{t} \widehat{\lambda}_{L L L X X}(t ; z)\right\}, \widehat{S}_{X L \leq X ; z}=\exp \left\{-\int_{0}^{t} \widehat{\lambda}_{X \mid L \leq X}(t ; z)\right\}$, where the cumulative hazard estimates are obtained from the corresponding Aalen's models. We further estimate $\widehat{b}(t ; z)=\widehat{S}_{X \mid L \leq X}(t ; z)-\widehat{S}_{L L L X X}(t ; z)$, and then the adjusted weight has the form

$$
\widetilde{w}_{i}^{*}(t)=\frac{r_{i}(t) \widehat{S}_{T}\left(X_{i}^{-} \wedge t^{-} ; Z_{i}\right) \widehat{b}(t)}{\widehat{b}\left(X_{i} \wedge t ; Z_{i}\right) \widehat{S}\left(t^{-}\right)} .
$$

Using the regression model-based weight, the asymptotic expression of the weighted score function can be extended similarly, which contains some complex terms. The simple variance estimation method may be considered.

\section{Simulation studies}

We considered two simulation studies. For the first simulation study, the weight function is assumed to be independent of the covariates. We examine the performances of two nonparametric weight estimators. In the second simulation study, we simulated data similar to the BMT example data analyzed in Section 5, for which the weight function depended on a binary covariate. We compared the performances of a stratified nonparametric weight estimator with a non-stratified weight estimator.

\subsection{Study 1}

The underlying regression models include two covariates. Given covariate values $z_{1}$ and $z_{2}$, the cumulative incidence functions are given by

$$
F_{1}\left(t ; z_{1}, z_{2}\right)=1-\left\{1-p\left(1-\mathrm{e}^{-\gamma_{1} t}\right)\right\}^{\exp \left(\beta_{11} z_{1}+\beta_{12} z_{2}\right)}
$$

and

$$
F_{2}\left(t ; z_{1}, z_{2}\right)=(1-p)^{\exp \left(\beta_{11} z_{1}+\beta_{12} z_{2}\right)} \times\left\{1-\mathrm{e}^{-\gamma_{2} t \exp \left(\beta_{21} z_{1}+\beta_{22} z_{2}\right)}\right\} .
$$

The covariate effect on the cumulative incidence function of cause 1 can be assessed via a proportional subdistribution hazards model. $\gamma_{1}$ and $\gamma_{2}$ were set at 0.7 and 0.5 , respectively. We let $p=0.7$ to generate settings with a dominant risk, and $p=0.5$ for settings with 
roughly equivalent risks. We considered both continuous covariates and discrete covariates. The continuous covariates $Z_{1}$ and $Z_{2}$ were generated from a standard normal distribution, and we set $\left(\beta_{11}, \beta_{12}\right)=(0.5,-0.5)$ and $\left(\beta_{21}, \beta_{22}\right)=(0.5,0.5)$. The discrete covariates $Z_{1}$ and $Z_{2}$ were generated from a Bernoulli distribution with equal probability to fall on 1 or 0 , and we set $\left(\beta_{11}, \beta_{12}\right)=(1,1)$ and $\left(\beta_{21}, \beta_{22}\right)=(-1,1)$.

In our simulation, the failure time is independent of the censoring and truncation time. Regarding $C$ and $L$, they can be independent or $C$ depends on $L$. Both were considered in our simulation to illustrate the difference in performance between weight 1 and 2 . For the settings with independent $C$ and $L$, the truncation time was generated from an exponential distribution with a hazard rate $\gamma_{l}$, and the censoring time was generated from a Uniform distribution in the interval $[a, b]$. A simulated observation would be discarded if either $T<L$ or $C<L$. In order to obtain a sample with size $n$, a larger number of realizations of ( $T, C, L$, $\left.Z_{1}, Z_{2}\right)$ need to be generated. The truncation rate is the percentage of the discarded observations out of all generated realizations. The censoring rate is the percentage of the censored observations out of $n$, based on the observed sample. In this study, we considered two levels of truncation rate ( 25 and 50 per cent) and three levels of the censoring rate $(0,25$ and 50 per cent). Values of $\gamma_{l}, a$ and $b$ were selected so that the average truncation rate and censoring rate, based on 1000 replicates, coincided with the predetermined rates.

For the settings in which $C$ depends on $L$, the left truncation time was still generated from an exponential distribution, and $\widetilde{C}$ was generated from $N\left(\mu, \sigma^{2}\right)$. To obtain the predetermined censoring and truncation rates, $\mu$ and $\sigma$ vary in the ranges 0.6-2.9 and 0.3-0.9, respectively. If $T \leq L$ or $\widetilde{C}<0$, the observation would be discarded; if not, we let the censoring time to be the sum of $L$ and $\widetilde{C}$. $T$ would be censored if it is greater than the censoring time.

For each setting, we simulated 1000 replicates with $n=200$. The regression coefficients $\boldsymbol{\beta}_{11}$ and $\boldsymbol{\beta}_{12}$ should be estimated by the methods described in Section 3.3. We report the average estimated regression coefficients, $E(\widehat{\beta})$, the sample standard deviation of $\widehat{\beta}, \operatorname{SD}(\widehat{\beta})$ and the average of estimated standard error, $E(\widehat{\mathrm{se}})$. Tables I and II show the simulation results.

It can be concluded from the above tables that the regression parameter estimates using the proposed weight estimators are very close to the true values under all the settings considered in this simulation study. For most of the settings, $\widehat{\beta}^{(1)}$ and $\widehat{\beta}^{(2)}$ yield indistinguishable results. Only with highly truncated settings, $\widehat{\beta}^{(1)}$ departs slightly more from the true value than $\widehat{\beta}^{(2)}$. The proposed variance estimators seem to satisfactorily measure the variations of the regression parameter estimates. $\widehat{\Sigma}_{\beta}^{(2)}$ gives well performance with discrete covariates, but slightly overestimates the true variance with continuous covariates. For the settings we considered, $\widehat{\Sigma}_{\beta}^{(1)}$ slightly underestimates the true variance. It can be observed that, in all the settings, the standard errors of $\widehat{\beta}^{(2)}$ are noticeably higher than those of $\widehat{\beta}^{(1)}$. This is due to the utilization of a stabilized weight for $\widehat{\beta}^{(1)}$ as suggested by Robins and Finkelstein [13], as well as Robins et al. [14]. Weight 2 used for $\widehat{\beta}^{(2)}$ is an unstabilized weight. A stabilized form for weight 2 is provided in Remark 4 of Section 3.2. We conducted a limited simulation to compare estimation performances between weight 2 and modified weight 2 . Table III shows the simulation results, revealing that, for the modified weight, the variation among regression coefficient estimates is greatly reduced, but the bias increases. Further study is needed to investigate the proper stabilized form for weight 2. 


\subsection{Study 2}

We conducted a simulation study to investigate the problem of dependent censoring and truncation, as well as the performance of the covariate adjusted weight. We generated a single binary covariate and let the underlying cumulative incidence functions to be

$$
F_{1}(t ; z)=1-\left\{1-0.7\left(1-\mathrm{e}^{-0.5 t}\right)\right\}^{\exp \left(\beta_{1} z\right)}
$$

and

$$
F_{2}(t ; z)=(1-0.7)^{\exp \left(\beta_{1} z\right)}\left\{1-\mathrm{e}^{-0.5 \exp \left(\beta_{2} z\right) t}\right\}
$$

The censoring time and truncation time were generated from exponential distributions with the following hazard functions:

$$
\lambda_{C}(t ; z)=0.4 \exp \left(\beta_{C} z\right) \quad \text { and } \quad \lambda_{L}(t ; z)=1.8 \exp \left(\beta_{L} z\right) .
$$

$\beta_{C}$ and $\beta_{L}$ have been set to different values for the first four settings. For the last setting, we generated the truncation times for $z=1$ group only, which is similar to the BMT example data considered in Section 5.

Both the nonparametric weight estimator given in equation (5) and its stratified version have been implemented on the simulated settings. The simulation result given in Table IV shows that the stratified weight performed very well for the settings with dependence between the covariate and the truncation time, as well as the censoring time. The results based on the nonparametric weight are acceptable although the required assumptions are not fully satisfied. For the stratified weight, the estimated standard errors based on the simple variance estimation method are very close to the degree of the variability among the regression parameter estimates. It is practically feasible to employ this method to obtain a computationally efficient estimator.

\section{A real example}

Chemotherapy should be administered to children with relapsed leukemia until they achieve their second remission. There are two options to continuously treat children in their second remission: chemotherapy or BMT. Barrett et al. [1] conducted a study to compare effectiveness of these two treatments on the LFS. The response variable of their study was the time from the beginning of second remission to either leukemia relapse or TRM. The study cohort consisted of reported cases from two sources, the International Bone Marrow Transplant Registry (IBMTR) and the Pediatric Oncology Group (POG). The IBMTR cohort consists of 376 children who received transplantation in second complete remission, and the POG cohort collects 540 children who had been continuously treated by chemotherapy. It should be noted that the patients who died while waiting for BMT were not reported to IBMTR. Thus, the survival times of the children in the transplantation group were left truncated by the transplantation times. Meanwhile, the survival time in both groups was subject to right censoring. The censoring rate is 29 per cent for the BMT, and 44 per cent for the chemotherapy group. The end of the study was the major reason of censoring.

The goal of our study was to compare two treatments on the cumulative incidences of leukemia relapse and TRM, respectively, adjusting for significant risk factors. We considered the following risk factors: sex ( 0 if female; 1 if male), age ( 0 if $\leq 10$ years; 1 if 
$>10$ years), leukocyte count at diagnosis ( 0 if $\leq 100000$ cells $/ \mathrm{mm}^{3} ; 1$ if $>100000$ cells $/ \mathrm{mm}^{3}$ ), the T-cell phenotype ( 0 if no; 1 if yes), duration of the first remission ( 0 if $\leq 18$ months; 1 if $>18$ months), and year of diagnosis ( 0 if before 1984; 1 if after 1984). For each competing risk, we constructed both the proportional cause-specific hazards model and the proportional subdistribution hazards model. A forward stepwise selection procedure was used to identify significant risk factors with criterion 0.05 .

For this example, the nonparametric weight is not appropriate since left truncation is present only in the transplantation group, thus, the truncation time is associated with the covariate for the treatment group. We utilized the stratified weight discussed in Section 3.4, with treatment groups serving as strata. The results of model construction for leukemia relapse are given in Table V. For the model on the cause-specific hazard, duration of the first remission (DCR) and the T-cell phenotype were identified as significant risk factors. However, DCR was the only risk factor significantly associated with the subdistribution hazard. For TRM (see Table VI), T-cell phenotype, year of diagnosis (DXYR84) and sex (Male) were identified as significant risk factors for both models. Age was marginally significant for the model on the cause-specific hazard, but was not significant for the model on the subdistribution hazard.

The predicted cumulative incidence curves of leukemia relapse for two treatments are given in Figure 1, where the significant risk factor, duration of the first remission, is set at $>18$ months. A 95 per cent log-log transformed pointwise confidence interval is also plotted in the figure. Figure 2 gives the predicted cumulative incidence curves of TRM for a boy without the T-cell phenotype and diagnosed after 1984, together with a 95 per cent log-log transformed confidence interval.

\section{Concluding remarks}

In this paper, we have extended the proportional subdistribution hazards model to the rightcensored and left-truncated competing risks data. The crucial adjustment with truncated data is to adopt the reciprocal of the truncation-censoring probability $G_{L, C}\left(X_{i} \wedge t Z_{i}\right)$ as the weight. When the weight does not depend on the covariates $\boldsymbol{Z}$, we proposed two nonparametric estimators for $G_{L, C}\left(X_{i} \wedge t\right)$. The first estimator uses the estimate of the survival probability of the all-cause failure time $T$ and the second estimator includes the estimate of $P(C-L>t)$. We have shown that the first weight estimator can be fully explained by the mass of a subject at time $t$ [10] and leads to the Aalen-Johansen estimator of the cumulative incidence function, yet the second weight estimator does not have this property.

For the first weight estimator, the stabilized version was proposed and utilized, which causes a noticeably lower degree of the variability in the regression parameter estimates. Further study is needed to improve the second weight estimator and hence reduce the variability of the regression parameter estimates.

The covariate adjusted weight can be potentially adopted. We have shown in this paper that the stratified nonparametric weight is a proper solution when the censoring/truncation time is associated with some discrete covariates. One may consider the covariate adjusted weight based on some regression models. However, it is difficult to choose proper models when certain type of the subdistribution hazard model is given. Such a problem has been indirectly addressed by Latouche et al. [16] by clarifying that a proportional subdistribution hazards model does not coexist with a proportional cause-specific hazards model. We suggested using Aalen's model and further study in this direction is needed. 


\section{Acknowledgments}

Dr Mei-Jie Zhang's research was supported by National Cancer Institute grant RO1 CA54706-13.

\section{Appendix A}

Here, we show that $G_{L, C}(X \wedge d \mid Z)=b(X \wedge d Z) / S\left(X^{-} \wedge t \mid Z\right)$ under the assumption that $T$ is independent of $L$ and $C$ given covariates $Z$. Since

$$
\begin{aligned}
b(t \mid Z) & =P\{L \leq t \leq(T \wedge C), L \leq(T \wedge C) \mid Z\} / P(L \leq X \mid Z) \\
& =P(T \geq t, L \leq t \leq C \mid Z) / P(L \leq X \mid Z) \\
& =P(T \geq t \mid Z) \times P(L \leq t \leq C \mid Z) / P(L \leq X \mid Z)
\end{aligned}
$$

it follows that

$$
\begin{aligned}
b(X \wedge t \mid Z) & =S\left(X^{-} \wedge t^{-} \mid Z\right) \times P(L \leq(X \wedge t) \leq C \mid Z) / P(L \leq X \mid Z) \\
& =S\left(X^{-} \wedge t^{-} \mid Z\right) \times P\{L \leq(X \wedge t) \leq C \mid L \leq X, Z\} \\
& =S\left(X^{-} \wedge t^{-} \mid Z\right) G_{L, C}(X \wedge t \mid Z) .
\end{aligned}
$$

Next, we show that $G_{L, C}(X \wedge t \mid Z)=G_{\widetilde{C} \tilde{X} \geq 0}\{(X \wedge t)-L \mid Z\} \times K(X \wedge t \mid Z)$, where $G_{\widetilde{C} \widetilde{X} \geq 0}(t \mid Z)=P(\widetilde{C} \geq t \mid \widetilde{X} \geq 0, t \geq 0, Z)$ and $K(t \mid Z)=P(L \leq \mid L \leq X, \boldsymbol{Z})$. For the left-truncated and right-censored data, individuals are observed only if $L \leq X$, which is equivalent to $\widetilde{X}=X-L \geq 0$. It follows that

$$
\begin{aligned}
P(L \leq(X \wedge t) \leq & C \mid L \leq X, Z) \\
& =P(0 \leq[(X \wedge t)-L] \leq \widetilde{C} \mid \widetilde{X} \geq 0, Z) \\
& =P(\widetilde{C} \geq[(X \wedge t)-L], L \leq(X \wedge t) \mid \widetilde{X} \geq 0, Z) \\
& =P(\widetilde{C} \geq[(X \wedge t)-L] \mid(X \wedge t)-L \geq 0, \widetilde{X} \geq 0, Z) \times P(L \leq(X \wedge t) \mid L \leq X, Z) .
\end{aligned}
$$

\section{Appendix B: Weak convergence of $n-1 / 2 U(k)(\beta 0)$}

For $k=1,2$, define the following notations:

$$
\begin{aligned}
\boldsymbol{S}_{k}^{(p)} & =n^{-1} \sum_{i=1}^{n} \widehat{w}_{i}^{(k)}(t) Y_{i}^{L, 1}(t) Z_{i}^{\otimes p} \exp \left(\boldsymbol{Z}_{i}^{\mathrm{T}} \beta\right) \quad \forall p=0,1,2, \\
\boldsymbol{I}^{(k)}(\beta) & =\left(-\frac{\partial \boldsymbol{U}^{(k)}(\beta)}{\partial \beta}\right)=\sum_{i=1}^{n} \int_{0}^{\infty}\left\{\frac{\boldsymbol{S}_{k}^{(2)}(\beta, t)}{S_{k}^{(0)}(\beta, t)}-\left(\frac{\boldsymbol{S}_{k}^{(1)}(\beta, t)}{S_{k}^{(0)}(\beta, t)}\right)^{\otimes 2}\right\} \mathrm{d}\left\{\widehat{w}_{i}^{(k)}(t) N_{i}^{1}(t)\right\} .
\end{aligned}
$$

Let

$$
\begin{aligned}
\boldsymbol{s}_{k}^{(p)}(\beta, u) & =\lim _{n \rightarrow \infty} \boldsymbol{S}_{k}^{(p)}(\beta, u) \quad \forall p=0,1,2, \\
\Omega^{(k)} & =\lim _{n \rightarrow \infty} n^{-1} \boldsymbol{I}^{(k)}(\beta) .
\end{aligned}
$$

Taking a Taylor expansion on $U^{(k)}(\boldsymbol{\beta})$ around $U\left(\boldsymbol{\beta}_{0}\right)$, we obtain 


$$
n^{1 / 2}\left(\widehat{\beta}-\beta_{0}\right) \approx_{\mathscr{P}}\left(\Omega^{(k)}\right)^{-1} n^{-1 / 2} \boldsymbol{U}^{(k)}\left(\beta_{0}\right)
$$

Considering weight 1 and following Fine and Gray's [7] arguments, we have

$$
\begin{aligned}
n^{-1 / 2} \boldsymbol{U}^{(1)}\left(\beta_{0}\right) \approx_{\mathscr{P}} & n^{-1 / 2} \sum_{i=1}^{n} \int_{0}^{\infty}\left\{Z_{i}(u)-\frac{s_{1}^{(1)}\left(\beta_{0}, u\right)}{s_{1}^{(0)}\left(\beta_{0}, u\right)}\right\} w_{i}^{(1)}(u) \mathrm{d} M_{i}^{L, 1}(u) \\
& +n^{-1 / 2} \sum_{i=1}^{n} \int_{0}^{\infty}\left\{Z_{i}(u)-\frac{\boldsymbol{s}^{(1)}\left(\beta_{0}, u\right)}{s_{1}^{(0)}\left(\beta_{0}, u\right)}\right\} \frac{\widehat{b}(u)}{\bar{b}\left(X_{i} \wedge u\right)}\left\{\frac{\widehat{S}\left(X_{i}^{-} \wedge u^{-}\right)}{\widehat{S}\left(u^{-}\right)}-\frac{S\left(X_{i}^{-} \wedge u^{-}\right)}{S\left(u^{-}\right)}\right\} \\
& \times r_{i}(u) \mathrm{d} M_{i}^{L, 1}(u) \\
& +n^{-1 / 2} \sum_{i=1}^{n} \int_{0}^{\infty}\left\{Z_{i}(u)-\frac{s_{1}^{(1)}\left(\beta_{0}, u\right)}{s_{1}^{(0)}\left(\beta_{0}, u\right)}\right\} \frac{S\left(X_{i}^{-} \wedge u^{-}\right)}{S\left(u^{-}\right)}\left\{\frac{\widehat{b}(u)}{\widehat{b}\left(X_{i} \wedge u\right)}-\frac{b(u)}{b\left(X_{i} \wedge u\right)}\right\} \\
& \times r_{i}(u) \mathrm{d} M_{i}^{L, 1(u)},
\end{aligned}
$$

where $w_{i}^{(1)}(t)=r_{i}(t) G_{L, C}(t) / G_{L, C}\left(X_{i} \wedge t\right)$ and $M_{i}^{L, 1}(t)=N_{i}^{L, 1}(t)-\int_{0}^{t} Y_{i}^{L, 1}(u) \lambda_{10}^{*}(u) \exp \left(Z_{i}^{\mathrm{T}} \beta_{0}\right) \mathrm{d} u$ is a martingale.

The first term is the main term, whereas the second and third terms account for the influence due to random weight. The left-truncated Kaplan-Meier estimator in the second term can be further expressed by

$$
\begin{aligned}
\frac{\widehat{S}\left(X_{i}^{-} \wedge t^{-}\right)}{\widehat{S}\left(t^{-}\right)} & =I\left(X_{i}<t\right)\left\{\frac{\widehat{S}\left(X_{i}^{-}\right)-S\left(X_{i}^{-}\right)}{\widehat{S}\left(t^{-}\right)}+S\left(X_{i}^{-}\right)\left(\frac{1}{\widehat{S}\left(t^{-}\right)}-\frac{1}{S\left(t^{-}\right)}\right)\right\} \\
& \approx p \quad \frac{S\left(X_{i}^{-}\right)}{S\left(t^{-}\right)} \sum_{j=1}^{n} \int_{0}^{\infty} \frac{I\left(X_{i}<u<t\right) \mathrm{d} M_{j \bullet}(u)}{\sum_{k=1}^{n} I\left(L_{k} \leq u \leq X_{k}\right)},
\end{aligned}
$$

where $M_{j \bullet}(t)=I\left\{X_{j} \leq t, \varepsilon_{j} \in(1,2)\right\}-\int_{0}^{t} I\left(L_{j} \leq u \leq X_{j}\right) \lambda(u) \mathrm{d} u$ is the martingale with respect to the self-exciting filtration (see [17, pp. 433 and 434]), and $\lambda(u)$ is the hazard rate for the failure of all causes.

For the third term,

$$
\begin{aligned}
\frac{\widehat{b}(t)}{\widehat{b}\left(X_{i} \wedge t\right)}-\frac{b(t)}{b\left(X_{i} \wedge t\right)}= & I\left(X_{i}<t\right)\left[\frac{1}{\widehat{b}\left(X_{i}\right)}\{\widehat{b}(t)-b(t)\}+b(t)\left\{\frac{1}{\widehat{b}\left(X_{i}\right)}-\frac{1}{b\left(X_{i}\right)}\right\}\right] \\
\approx_{\mathscr{P}} & \frac{I\left(X_{i}<t\right.}{\left.n b\left(X_{i}\right)\right)} \sum_{j=1}^{n}\left\{I\left(L_{j} \leq t \leq X_{j}\right)-b(t)\right\} \\
& +\frac{I\left(x_{i}<t\right) b(t)}{n b\left(X_{i}\right)^{2}} \sum_{j=1}^{n}\left\{b\left(X_{i}\right)-I\left(L_{j} \leq X_{i} \leq X_{j}\right)\right\} \\
= & \frac{I\left(X_{i}<t\right) b(t)}{n b\left(X_{i}\right)} \sum_{j=1}^{n}\left\{\frac{1}{b(t)} I\left(L_{j} \leq t \leq X_{j}\right)-\frac{1}{b\left(X_{i}\right)} I\left(L_{j} \leq X_{i} \leq X_{j}\right)\right\} .
\end{aligned}
$$

Summarizing the above derivations, we obtain the asymptotically equivalent expression of $n^{-1 / 2} U^{(1)}\left(\beta_{0}\right)$

$$
n^{-1 / 2} \boldsymbol{U}^{(1)}\left(\beta_{0}\right) \approx n^{-1 / 2} \boldsymbol{W}_{\beta, i}^{(1)} \approx n^{-1 / 2} \sum_{i=1}^{n}\left\{\phi_{i}+\chi_{i}+\psi_{i}\right\}
$$


where

$$
\begin{aligned}
\phi_{i}= & \int_{0}^{\infty}\left\{Z_{i}-\frac{s_{1}^{(1)}\left(\beta_{0}, t\right)}{s_{1}^{(0)}\left(\beta_{0}, t\right)}\right\} w_{i}^{(1)}(t) \mathrm{d} M_{i}^{L, 1}(t), \\
\chi_{i}= & \int_{0}^{\infty} \frac{\boldsymbol{q}(u)}{\pi(u)} \mathrm{d} M_{i \bullet}(u), \\
\boldsymbol{q}(u)= & \lim _{n \rightarrow \infty} n^{-1} \sum_{j=1}^{n} \int_{0}^{\infty}\left\{Z_{j}-\frac{\boldsymbol{s}_{1}^{(1)}\left(\beta_{0}, s\right)}{s_{1}^{(0)}\left(\beta_{0}, s\right)}\right\} w_{j}^{(1)}(s) \mathrm{d} M_{j}^{L, 1}(s) I\left(X_{j}<u<s\right), \\
\pi(u)= & \lim _{n \rightarrow \infty} n^{-1} \sum_{j=1}^{n} I\left(L_{j} \leq u \leq X_{j}\right), \\
\psi_{i}= & \lim _{n \rightarrow \infty} n^{-1} \sum_{j=1}^{n} \int_{0}^{\infty}\left\{Z_{j}-\frac{s_{1}^{(1)}\left(\beta_{0}, s\right)}{s_{1}^{(0)}\left(\beta_{0}, s\right)}\right\} w_{j}^{(1)}(s) \mathrm{d} M_{j}^{L, 1}(s) I\left(X_{j}<s\right) \\
& \times\left\{\frac{I\left(L_{i} \leq s \leq X_{i}\right)}{b(s)}-\frac{I\left(L_{i} \leq X_{j} \leq X_{i}\right)}{b\left(X_{j}\right)}\right\} .
\end{aligned}
$$

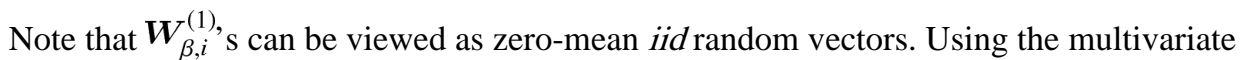
central limit theorem, $n^{-1 / 2} U^{(1)}\left(\boldsymbol{\beta}_{0}\right)$ converges in distribution to a zero-mean Gaussian random vector and the covariance matrix can be consistently estimated by

$$
n^{-1} \sum_{i}\left(\widehat{\boldsymbol{W}}_{\beta, i}^{(1)}\right)^{\otimes 2}
$$

where

$$
\begin{aligned}
\widehat{\boldsymbol{W}}_{\widehat{\beta}, i}^{(1)}= & \widehat{\phi}_{i}+\widehat{\chi}_{i}+\widehat{\psi}_{i}, \\
\widehat{\phi}_{i}= & \int_{0}^{\infty}\left\{Z_{i}-\frac{S_{1}^{(1)}\left(\widehat{(}^{(1)}, t\right)}{S_{1}^{(0)}\left(\widehat{\beta}^{(1)}, t\right)}\right\} \widehat{w}_{i}^{(1)}(t) \mathrm{d} \widehat{M}_{i}^{L, 1}(t), \\
\widehat{\chi}_{i}= & \int_{0}^{\infty} \widehat{\widehat{q}(u)} \overline{\bar{\pi}(u)} \mathrm{d} \widehat{M}_{i \bullet}(u), \\
\widehat{\boldsymbol{q}}(u)= & n^{-1} \sum_{i} \int_{0}^{\infty}\left\{Z_{i}-\frac{S_{1}^{(1)}\left(\widehat{\beta}^{(1)}, s\right)}{S_{1}^{(0)}\left(\widehat{\beta}^{(1)}, s\right)}\right\} \widehat{w}_{i}^{(1)}(s) \mathrm{d} \widehat{M}_{i}^{L, 1}(s) I\left(X_{j}<u<s\right), \\
\widehat{\pi}(u)= & n^{-1} \sum_{i} I\left(L_{i} \leq u \leq X_{i}\right), \\
\widehat{\psi}_{i}= & n^{-1} \sum_{j} \int_{0}^{\infty}\left\{Z_{j}-\frac{S^{(1)}\left(\widehat{\beta}^{(1)}, s\right)}{S^{(0)}\left(\widehat{\beta}^{(1)}, s\right)}\right\} \widehat{w}_{j}^{(1)}(s) \mathrm{d} \widehat{M}_{j}^{L, 1}(s) I\left(X_{j}<s\right) \\
& \times\left\{\frac{I\left(L_{i} \leq s \leq X_{i}\right)}{\widehat{b}(s)}-\frac{I\left(L_{i} \leq X_{j} \leq X_{i}\right)}{\widehat{b}\left(X_{j}\right)}\right\}, \\
\widehat{M}_{j \bullet}(t)= & I\left(X_{j} \leq t, \varepsilon_{j} \in\{1,2\}\right)-\int_{0}^{t} I\left(L_{j} \leq u \leq X_{j}\right) d \widehat{\Lambda}(u), \\
\widehat{\Lambda}(t)= & \sum_{i} \int_{0}^{t} \frac{\mathrm{d}\left(X_{i} \leq u, \varepsilon_{i} \in\{1,2\}\right)}{\sum_{j} I\left(L_{j} \leq u \leq X_{j}\right)} .
\end{aligned}
$$

When utilizing weight 2 , we can similarly show that $n^{-1 / 2} U^{(2)}\left(\boldsymbol{\beta}_{0}\right)$ is asymptotically equivalent to $n^{-1 / 2} \boldsymbol{W}_{\beta, i}^{(2)}$, which has a zero-mean normal distribution and the covariance matrix can be consistently estimated by

$$
n^{-1} \sum_{i}\left(\widehat{\boldsymbol{W}}_{\beta, i}^{(2)}\right)^{\otimes 2}
$$

where 


$$
\begin{aligned}
\widehat{\boldsymbol{W}}_{\beta, i}^{(2)} & =\widehat{\gamma}_{i}+\widehat{\eta}_{i}+\widehat{\theta}_{i}, \\
\widehat{\gamma}_{i} & =\int_{0}^{\infty}\left\{\boldsymbol{Z}_{i}-\frac{\boldsymbol{S}_{2}^{(1)}\left(\widehat{\beta}^{(2)}, t\right)}{S_{2}^{(0)}\left(\widehat{\beta}^{(2)}, t\right)}\right\} \widehat{w}_{i}^{(2)}(t) \mathrm{d} \widehat{M}_{i}^{L, 1}(t), \\
\widehat{\eta}_{i} & =\int_{0}^{\infty} \widehat{\bar{\alpha}(u)} \overline{\bar{\pi}(u)} \mathrm{d} \widehat{M}_{i}^{c}(u), \\
\widehat{\alpha}(u) & =n^{-1} \sum_{j=1}^{n} \int_{0}^{\infty}\left\{Z_{i}-\frac{S_{2}^{(1)}\left(\widehat{\beta}^{(2)}, s\right)}{S_{2}^{(0)}\left(\widehat{\beta}^{(2)}, s\right)}\right\} \widehat{w}_{j}^{(2)}(s) \mathrm{d} \widehat{M}_{j}^{L, 1}(s) I\left(u \leq\left\{\left(X_{j} \wedge s\right)-L_{j}\right\}\right), \\
\widehat{\theta}_{i} & =n^{-1} \sum_{j=1}^{n} \int_{0}^{\infty} \int_{0}^{\infty}\left\{Z_{i}-\frac{S_{2}^{(1)}\left(\widehat{\beta}^{(2)}, s\right)}{S_{2}^{(0)}\left(\widehat{\beta}^{2)}, s\right)}\right\} \widehat{w}_{j}^{(2)}(s) \mathrm{d} \widehat{M}_{j}^{L, 1}(s)\left\{1-\frac{I\left(L_{i} \leq\left(X_{j} \wedge s\right)\right)}{\widehat{K}\left(X_{j} \wedge s\right)}\right\}, \\
\widehat{w}_{i}^{(2)}(t) \widehat{M}_{i}^{L, 1}(t) & =\widehat{w}_{i}^{(2)}(t) N_{i}^{L, 1}(t)-\int_{0}^{t} \widehat{w}_{i}^{(2)}(u) Y_{i}^{L, 1}(u) \exp \left(Z_{i}^{\mathrm{T}} \widehat{\beta}^{(2)}\right) \mathrm{d} \widehat{\Lambda}_{10}^{*(2)}(u), \\
\widehat{M}_{j}^{c}(t) & =I\left(X_{j}-L_{j} \leq t, \varepsilon_{j}=0\right)-\int_{0}^{t} I\left(X_{j}-L_{j} \geq u\right) \mathrm{d} \widehat{\Lambda}_{c}(u), \\
\widehat{\Lambda}_{c}(t) & =\sum_{i} \int_{0}^{t} \frac{\mathrm{d}\left(X_{i}-L_{i} \leq u, \varepsilon_{i}=0\right)}{\sum_{j} I\left(X_{j}-L_{j} \geq u\right)} .
\end{aligned}
$$

\section{Appendix C: Weak convergence of $n 1 / 2\left\{\Lambda^{\wedge} 10 *(k)-\Lambda 10 *(t)\right\}$}

By Taylor expansion

$$
\begin{array}{ll} 
& n^{1 / 2}\left\{\widehat{\Lambda}_{10}^{*(1)}(t)-\Lambda_{10}^{*}(t)\right\} \\
\approx_{\mathscr{P}} & n^{-1 / 2} \sum_{i=1}^{n} \int_{0}^{t} \frac{\widehat{w}_{i}^{(1)}(u) \mathrm{d} M_{i}^{L, 1}(u)}{s_{1}^{(0)}\left(\beta_{0}, u\right)}-\int_{0}^{t} \frac{\left\{s_{1}^{(1)}\left(\beta_{0}, u\right)\right\}^{\mathrm{T}}}{s_{1}^{(0)}\left(\beta_{0}, u\right)} \mathrm{d} \Lambda_{10}^{*}(u) \sqrt{n}\left\{\widehat{\beta}^{(1)}-\beta_{0}\right\} \\
\approx_{\mathscr{P}} & n^{-1 / 2} \sum_{i=1}^{n} \int_{0}^{t} \frac{w_{i}(u) \mathrm{d} M_{i}^{L, 1}(u)}{s_{1}^{(0)}\left(\beta_{0}, u\right)}-\int_{0}^{t} \frac{\left\{s_{1}^{(1)}\left(\beta_{0}, u\right)\right\}^{\mathrm{T}}}{s_{1}^{(0)}\left(\beta_{0}, u\right)} \mathrm{d} \Lambda_{10}^{*}(u) \sqrt{n}\left\{\widehat{\beta}^{(1)}-\beta_{0}\right\} \\
& +n^{-1 / 2} \sum_{i} \int_{0}^{t} \frac{\left.\widehat{w}_{i}^{(1)}(u)-w_{i}(u)\right\}}{s_{1}^{(0)}\left(\beta_{0}, u\right)} \\
\approx_{\mathscr{P}}^{L, 1}(u) & n^{-1 / 2} \sum_{i} W_{\Lambda_{10}^{*}, i}^{(1)}(t),
\end{array}
$$

where

$$
\begin{aligned}
& W_{\Lambda_{10}^{*}, i}^{(1)}(t)=\int_{0}^{t} \frac{w_{i}(u) \mathrm{d} M_{i}^{L, 1}(u)}{s_{1}^{(0)}\left(\beta_{0}, u\right)} \\
& -\int_{0}^{t} \frac{\left\{s_{1}^{(1)}\left(\beta_{0}, u\right)\right\}^{\mathrm{T}}}{s_{1}^{(0)}\left(\beta_{0}, u\right)} \mathrm{d} \Lambda_{10}^{*}(u)\left(\Omega^{(1)}\right)^{-1}\left(\phi_{i}+\chi_{i}+\psi_{i}\right)+\int_{0}^{\infty} \frac{v(u, t)}{\pi(u)} \mathrm{d} M_{i \bullet}(u)+\mu_{i}(t) \\
& v(u, t)=\lim _{n \rightarrow \infty} n^{-1} \sum_{j=1}^{n} \int_{0}^{t} \frac{w_{j}^{(1)}(s) \mathrm{d} M_{j}^{L, 1}(s)}{s_{1}^{(0)}\left(\beta_{0}, s\right)} I\left(X_{j}<u<s\right), \\
& \mu_{i}(t)=\lim _{n \rightarrow \infty} n^{-1} \sum_{j=1}^{n} \int_{0}^{t} \frac{w_{j}^{(1)}(s) \mathrm{d} M_{j}^{L, 1}(s)}{s_{1}^{(0)}\left(\beta_{0}, s\right)} I\left(X_{j}<s\right)\left\{\frac{I\left(L_{i} \leq s \leq X_{i}\right)}{b(s)}-\frac{I\left(L_{i} \leq X_{j} \leq X_{i}\right)}{b\left(X_{j}\right)}\right\} .
\end{aligned}
$$

By the central limit theorem, $n^{1 / 2}\left\{\widehat{\Lambda}_{10}^{*(1)}-\Lambda_{10}^{*}(t)\right\}$ converges in finite-dimensional distribution to a zero-mean Gaussian process. Using the empirical theory as in Fine and Gray [7], and in Lin et al. [18], we can show that $n^{-1 / 2} W_{\Lambda_{10}^{*}, i}^{(1)}(t)$ is tight. Thus, $n^{1 / 2}\left\{\widehat{\Lambda}_{10}^{*(1)}-\Lambda_{10}^{*}(t)\right\}$ converges weakly to a zero-mean Gaussian process and its variance can be consistently estimated by

$$
\widehat{\Sigma}_{\Lambda_{10}^{*}}^{(1)}(t)=n^{-1} \sum_{i=1}^{n}\left\{\widehat{W}_{\Lambda_{10}^{*}, i}^{(1)}(t)\right\}^{2},
$$


where

$$
\begin{aligned}
& \widehat{W}_{\Lambda_{10}^{*}, i}^{(1)}(t)=\int_{0}^{t} \frac{\widehat{W}_{i}^{(1)}(u) \mathrm{d} \widehat{M}_{i}^{L, 1}(u)}{S_{1}^{(0)}\left(\widehat{\beta}^{(1)}, u\right)}-\int_{0}^{t} \frac{\left(S_{1}^{(1)}\left(\widehat{\beta}^{(1)}, u\right)\right)^{\mathrm{T}}}{\left.S_{1}^{(0)} \widehat{\beta}^{(1)}, u\right)} \mathrm{d} \widehat{\Lambda}_{10}^{*(1)}(u)\left\{n^{-1} \boldsymbol{I}^{(1)}\left(\widehat{\beta}^{(1)}\right)\right\}^{-1}\left(\widehat{\phi}_{i}+\widehat{\chi}_{i}+\widehat{\psi}_{i}\right) \\
& +\int_{0}^{\infty} \frac{\widehat{v}(u, t)}{\widehat{\pi}(u)} \mathrm{d} \widehat{M}_{i \bullet}(u)+\widehat{\mu}_{i}(t), \\
& \widehat{v}(u, t)=\sum_{j} \int_{0}^{t} \frac{\widehat{w}_{j}^{(1)}(s) \mathrm{d} \widehat{M}_{j}^{L, 1}(s)}{S_{1}^{(0)}\left(\widehat{\beta}^{(1)}, s\right)} I\left(X_{j}<u<s\right), \\
& \widehat{\mu}_{i}(t)=\sum_{j} \int_{0}^{t} \frac{\widehat{w}_{j}^{(1)}(s) \mathrm{d} \widehat{M}_{j}^{L, 1}(s)}{S_{1}^{(0)}\left(\widehat{\beta}^{(1)}, s\right)} I\left(X_{j}<s\right)\left\{\frac{I\left(L_{i} \leq s \leq X_{i}\right)}{\widehat{b}(s)}-\frac{I\left(L_{i} \leq X_{j} \leq X_{i}\right)}{\widehat{b}\left(X_{j}\right)}\right\} .
\end{aligned}
$$

Using weight 2 , it can be similarly shown that $n^{1 / 2}\left\{\widehat{\Lambda}_{10}^{*(2)}-\Lambda_{10}^{*}(t)\right\}$ converges weakly to a zero-mean Gaussian process and its variance can be consistently estimated by

$$
\begin{aligned}
& \widehat{\Sigma}_{\Lambda_{10}^{*}}^{(2)}(t)=n^{-1} \sum_{i=1}^{n}\left\{\widehat{W}_{\Lambda_{10}^{*}, i}^{(2)}(t)\right\}^{2} \\
& \widehat{W}_{\Lambda_{10}^{*}, i}^{(2)}(t)= \int_{0}^{t} \frac{\widehat{W}_{i}^{(2)}(u) \mathrm{d} \widehat{\mathrm{d}}_{i}^{L, 1}(u)}{S_{2}^{(0)}\left(\widehat{\beta}^{(2)}, u\right)}-\int_{0}^{t} \frac{\left(S_{2}^{(1)}\left(\widehat{\beta}^{(2)}, u\right)\right)^{\mathrm{T}}}{S_{2}^{(0)}\left(\widehat{\beta}^{(2)}, u\right)} \mathrm{d} \widehat{\Lambda}_{10}^{*(2)}(u)\left\{n^{-1} \boldsymbol{I}^{(2)}\left(\widehat{\beta}^{(2)}\right)\right\}^{-1}\left(\widehat{\gamma}_{i}+\widehat{\eta}_{i}+\widehat{\theta}_{i}\right) \\
&+\int_{0}^{\infty} \frac{\left.\widehat{\rho}^{(u, t)}\right)}{\widehat{\pi}(u)} \widehat{M}_{i}^{c}(u)+\widehat{\varphi}_{i}(t), \\
& \widehat{\rho}(u, t)= n^{-1} \sum_{j=1}^{n} \int_{0}^{t} \frac{\widehat{w}_{j}^{(2)}(s) \mathrm{d} \widehat{M}_{j}^{L, 1}(s)}{S_{2}^{(0)}\left(\widehat{\beta}^{(2)}, s\right)} I\left(u \leq\left\{\left(X_{j} \wedge s\right)-L_{j}\right\}\right), \\
& \widehat{\varphi}_{i}(t)= \sum_{j=1}^{n} \int_{0}^{t} \frac{\widehat{W}_{j}^{(2)}(s) \mathrm{d} \widehat{\bar{M}}_{j}^{L, 1}(s)}{S_{2}^{(0)}\left(\widehat{\beta}^{(2)}, s\right)}\left\{1-\frac{I\left(L_{i} \leq\left(X_{j} \wedge s\right)\right)}{\widehat{K}\left(X_{j} \wedge s\right)}\right\} .
\end{aligned}
$$

\section{Appendix D: Weak convergence of $n 1 / 2\left\{F^{\wedge} 1(k)(t ; z)-F 1(t ; z)\right\}$}

Utilizing the functional delta method

$$
\begin{aligned}
n^{1 / 2}\left\{\widehat{F}_{1}^{(1)}(t ; z)-F_{1}(t ; z)\right\} \approx_{\mathscr{P}} & n^{1 / 2}\left\{1-F_{1}(t ; z)\right\}\left\{\exp \left(z^{\mathrm{T}} \widehat{\beta}^{(1)}\right)-\exp \left(z^{\mathrm{T}} \beta_{0}\right)\right\} \widehat{\Lambda}_{10}^{*^{(1)}}(t) \\
& +n^{1 / 2}\left\{1-F_{1}(t ; z)\right\} \exp \left(z^{\mathrm{T}} \beta_{0}\right)\left\{\widehat{\Lambda}_{10}^{*(1)}(t)-\Lambda_{10}^{*}(t)\right\} .
\end{aligned}
$$

Similarly, if follows that $n^{1 / 2}\left\{\widehat{F}_{1}^{(1)}(t ; z)-F_{1}(t ; z)\right\}$ weakly converges to a zero-mean Gaussian process, and the variance can be consistently estimated by

$$
\widehat{\Sigma}_{F_{1}}^{(1)}(t)=n^{-1}\left\{1-\widehat{F}_{1}^{(1)}(t ; z)\right\}^{2} \sum_{i=1}^{n}\left\{\widehat{W}_{F_{1}, i}^{(1)}(t ; z)\right\}^{2}
$$

where

$$
\begin{aligned}
\widehat{W}_{F_{1}, i}^{(1)}(t ; z)= & \int_{0}^{t} \frac{\exp \left(z^{\mathrm{T}} \widehat{\beta}^{(1)}\right) \bar{w}_{i}^{(1)}(u) \mathrm{d} \widehat{M}_{i}^{L, 1}(u)}{S^{(0)}\left(\widehat{\beta}^{(1)}, u\right)}+\left\{\widehat{\boldsymbol{h}}^{(1)}(t ; z)\right\}^{\mathrm{T}}\left\{\widehat{\beta}^{(1)}\right\}^{-1}\left(\widehat{\phi}_{i}+\widehat{\chi}_{i}+\widehat{\psi}_{i}\right) \\
& +\int_{0}^{\infty} \frac{\exp \left(z^{\top} \bar{\beta}^{(1)}\right) \bar{v}(u, t)}{\widehat{\pi}^{(u)}} \mathrm{d} \widehat{M}_{i \bullet}(u)+\exp \left(z^{\mathrm{T}} \widehat{\beta}^{(1)}\right) \widehat{\mu}_{i}(t), \\
\widehat{\boldsymbol{h}}^{(1)}(t ; z)= & \int_{0}^{t}\left\{z-\frac{\boldsymbol{S}_{1}^{(1)}\left(\widehat{\beta}^{(1)}, u\right)}{S_{1}^{(0)}\left(\widehat{\beta}^{(1)}, u\right)}\right\} \exp \left(z^{\mathrm{T}} \widehat{\beta}^{(1)}\right) \mathrm{d} \widehat{\Lambda}_{10}^{*(1)}(u) .
\end{aligned}
$$


For weight 2 , the variance of the limiting distribution of $n^{1 / 2}\left\{\widehat{F}_{1}^{(2)}(t ; z)-F_{1}(t ; z)\right\}$ can be consistently estimated by

$$
\widehat{\Sigma}_{F_{1}}^{(2)}(t)=n^{-1}\left\{1-\widehat{F}_{1}^{(2)}(t ; z)\right\}^{2} \sum_{i=1}^{n}\left\{\widehat{W}_{F_{1}, i}^{(2)}(t ; z)\right\}^{2}
$$

where

$$
\begin{aligned}
& \widehat{W}_{F_{2}, i}^{(1)}(t ; z)=\int_{0}^{t} \frac{\exp \left(z^{\mathrm{T}} \widehat{\beta}^{(2)}\right) \widehat{w}_{i}^{(2)}(u) \mathrm{d} \widehat{M}_{i}^{L, 1}(u)}{S^{(0)}\left(\widehat{\beta}^{(2)}, u\right)}+\left\{\widehat{\boldsymbol{h}}^{(2)}(t ; z)\right\}^{\mathrm{T}}\left\{\widehat{\beta}^{(2)}\right\}^{-1}\left(\widehat{\gamma}_{i}+\widehat{\eta}_{i}+\widehat{\theta}_{i}\right) \\
& +\int_{0}^{\infty} \frac{\exp \left(z^{\mathrm{T}} \widehat{\beta}^{(2)}\right) \widehat{\rho}(u, t)}{\widehat{\pi}(u)} \mathrm{d} \widehat{M}_{i}^{c}(u)+\exp \left(z^{\mathrm{T}} \widehat{\beta}^{(2)}\right) \widehat{\varphi}_{i}(t), \\
& \widehat{\boldsymbol{h}}^{(2)}(t ; z)=\int_{0}^{t}\left\{z-\frac{\boldsymbol{S}_{2}^{(1)}\left(\widehat{\beta}^{(2)}, u\right)}{S_{2}^{(0)}\left(\widehat{\beta}^{(2)}, u\right)}\right\} \exp \left(z^{\mathrm{T}} \widehat{\beta}^{(2)}\right) \mathrm{d} \widehat{\Lambda}_{10}^{(2)}(u) .
\end{aligned}
$$

\section{Appendix E}

Let $\widetilde{\beta}$ be the solution to the score estimating equation using stratified weight (7). For simplicity, treating the weight function known, the variance of $n^{1 / 2}\{\widetilde{\beta}-\beta\}$ can be estimated by

$$
\widetilde{\Sigma}_{\beta}=\left\{n^{-1} \boldsymbol{I}^{*}(\widetilde{\beta})\right\}^{-1} \times\left\{n^{-1} \sum_{r=1}^{R} \sum_{i=1}^{n_{r}}\left(\widehat{v}_{r i}\right)^{\otimes 2}\right\} \times\left\{n^{-1} \boldsymbol{I}^{*}(\widetilde{\beta})\right\}^{-1},
$$

where

$$
\begin{aligned}
\boldsymbol{S}_{*}^{(p)}(\beta, t) & =n^{-1} \sum_{r=1}^{R} \sum_{i=1}^{n_{r}} \widetilde{w}_{r i}(t) Y_{r i}^{L, 1}(t) Z_{r i}^{\otimes p} \exp \left(Z_{r i}^{\mathrm{T}} \beta\right) \quad \forall p=0,1,2, \\
\boldsymbol{I}^{*}(\beta) & =\sum_{r=1}^{R} \sum_{i=1}^{n_{r}} \int_{0}^{\infty}\left\{\frac{\boldsymbol{S}_{*}^{(2)}(\beta, t)}{S_{*}^{(0)}(\beta, t)-\left(\frac{S_{*}^{(1)}(\beta, t)}{S_{*}^{(0)}(\beta, t)}\right)^{\otimes 2}}\right\} \mathrm{d}\left\{\widetilde{w}_{r i}(t) N_{r i}^{L, 1}(t)\right\}, \\
\widehat{v}_{r i} & =\int_{0}^{\infty}\left\{\boldsymbol{Z}_{r i}-\frac{\boldsymbol{S}_{*}^{(1)}\left(\widetilde{\beta}_{*}\right)}{S_{*}^{(0)}(\widetilde{\beta}, t)}\right\} \widetilde{w}_{r i}(t) \mathrm{d} \widehat{M}_{r i}^{L, 1}(t), \\
\widetilde{w}_{r i}(t) \widehat{M}_{r i}^{L, 1}(t) & =\widehat{w}_{r i}(t) N_{r i}^{L, 1}(t)-\int_{0}^{t} \widetilde{w}_{r i}(u) Y_{r i}^{L, 1}(u) \exp \left(Z_{r i}^{\mathrm{T}} \widetilde{\beta}\right) \mathrm{d} \widetilde{\Lambda}_{10}^{*}(u), \\
\widetilde{\Lambda}_{10}^{*}(t) & =\sum_{r=1}^{R} \sum_{i=1}^{n_{r}} \int_{0}^{t} \frac{\mathrm{d} \boldsymbol{I}\left(\boldsymbol{X}_{r i} \leq u, \varepsilon_{r i} \in\{1,2\}\right)}{\sum_{l=1}^{R} \sum_{j=1}^{n_{l}} I\left(L_{l j} \leq u \leq X_{l j}\right)} .
\end{aligned}
$$

Let $\widetilde{F}_{1}(t ; z)$ be the estimator of the cumulative incidence function given $z$. Similarly, we estimate the asymptotic variance of $\widetilde{F}_{1}(t ; z)$ by

$$
n^{-1}\left\{1-\widetilde{F}_{1}(t ; z)\right\}^{2} \sum_{r=1}^{R} \sum_{i=1}^{n_{r}}\left\{\widetilde{W}_{F_{1}, r i}(t ; z)\right\}^{2}
$$

where 


$$
\begin{aligned}
\widetilde{W}_{F_{1}, r i}(t ; z) & =\int_{0}^{t} \frac{\exp \left(z^{\mathrm{T}} \widetilde{\beta}\right) \widetilde{w}_{r i}(u) \mathrm{d} \widehat{M}_{r i}^{L, 1}(u)}{S_{*}^{(0)}(\widetilde{\beta}, u)}+\{\widetilde{\boldsymbol{h}}(t ; z)\}^{\mathrm{T}}\left\{n^{-1} \boldsymbol{I}^{*}(\widetilde{\beta})\right\}^{-1} \widehat{\boldsymbol{v}}_{r i}, \\
\widetilde{\boldsymbol{h}}(t ; z) & =\int_{0}^{t}\left\{z-\frac{\boldsymbol{S}_{*}^{(1)}(\widetilde{\beta}, u)}{S_{*}^{(0)}(\widetilde{\beta}, u)}\right\} \exp \left(z^{\mathrm{T}} \widetilde{\beta}\right) \mathrm{d} \widetilde{\Lambda}_{10}^{*}(u)
\end{aligned}
$$

\section{References}

1. Barrett AJ, Horowitz MM, Pollock BH, Zhang MJ, Bortin MM, Buchanan GR, Gamitta BM, Ochs J, Graham-Pole J, Rowlings RA, Rimm AA, Klein JP, Shuster JJ, Sobocinski KA, Gale RP. Bone marrow transplants from HLA-identical siblings as compared with chemotherapy for children with acute lymphoblastic leukaemia in second remission. The New England Journal of Medicine. 1994; 331:1253-1258. [PubMed: 7935682]

2. Cheng SC, Fine JP, Wei LJ. Prediction of cumulative incidence function under the proportional hazards model. Biometrics. 1998; 54:219-228. [PubMed: 9544517]

3. Shen Y, Cheng SC. Confidence bands for cumulative incidence curves under the additive risk model. Biometrics. 1999; 55:1093-1100. [PubMed: 11315053]

4. Scheike TH, Zhang MJ. An additive multiplicative Cox-Aalen regression model. Scandinavian Journal of Statistics. 2002; 29:75-88.

5. Scheike TH, Zhang MJ. Extensions and applications of the Cox-Aalen survival model. Biometrics. 2003; 59:1036-1045. [PubMed: 14969483]

6. Andersen, PK.; Borgan, O.; Gill, RD.; Keiding, N. Statistical Models Based on Counting Processes. Springer; New York: 1993.

7. Fine JP, Gray RJ. A proportional hazards model for the subdistribution of a competing risk. Journal of the American Statistical Association. 1999; 94:496-509.

8. Sun LQ, Liu JX, Sun JG, Zhang MJ. Modelling the subdistribution of a competing risk. Statistica Sinica. 2006; 16(4):1367-1385.

9. Scheike TH, Zhang MJ, Gerds TA. Predicting cumulative incidence probability by direct binomial regression. Biometrika. 2008; 95:205-220.

10. Zhang X, Zhang MJ, Fine JP. A mass redistribution algorithm for right-censored and left-truncated time to event data. Journal of Statistical Planning and Inference. 2009; 9:3329-3339. [PubMed: 22553383]

11. Efron, B. The two sample problem with censored data. Proceedings of the Fifth Berkeley Symposium on Mathematical Statistics and Probability; New York: Prentice-Hall; 1967. p. 831-853.

12. Robins, JM.; Rotnitzky, A. Recovery of information and adjustment for dependent censoring using surrogate markers. In: Jewell, N.; Dietz, K.; Farewell, V., editors. AIDS EpidemiologyMethodological Issues. Birkhauser; Boston: 1992. p. 297-331.

13. Robins JM, Finkelstein D. Correcting for non-compliance and dependent censoring in an aids clinical trial with inverse probability of censoring weighted (IPCW) log-rank tests. Biometrics. 2000; 56(3):779-788. [PubMed: 10985216]

14. Robins JM, Hernán M, Brumback B. Marginal structural models and causal inference in epidemiology. Epidemiology. 2000; 11(5):550-560. [PubMed: 10955408]

15. Andersen PK, Gill RD. Cox's regression model for counting processes: a large sample study. Annals of Statistics. 1982; 10:1100-1120.

16. Latouche A, Boisson V, Chevret S, Porcher R. Misspecified regression model for the subdistribution hazard of a competing risk. Statistics in Medicine. 2007; 26:965-974. [PubMed: 16755533]

17. Lai TL, Ying Z. Estimating a distribution function with truncated and censored data. Annals of Statistics. 1991; 19:417-442.

18. Lin DY, Wei LJ, Yang I, Ying Z. Semiparametric regression for the mean and rate functions of recurrent events. Journal of the Royal Statistical Society, B. 2000; 62:711-731. 


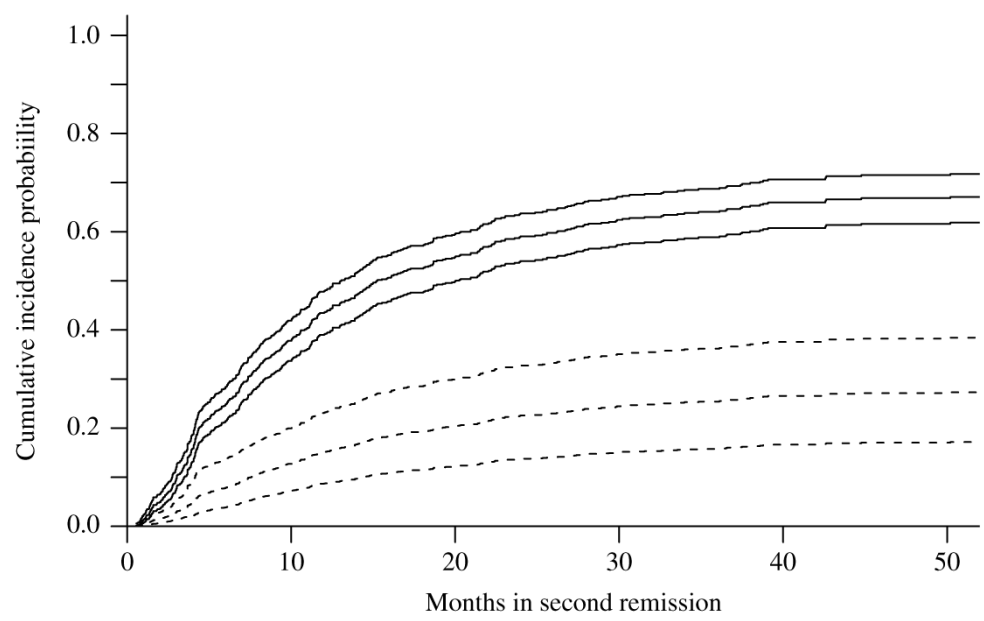

Figure 1.

Predicted cumulative incidences of leukemia relapse for chemotherapy (solid) and BMT (dashes) based on a child with duration of first remission $>18$ months. 


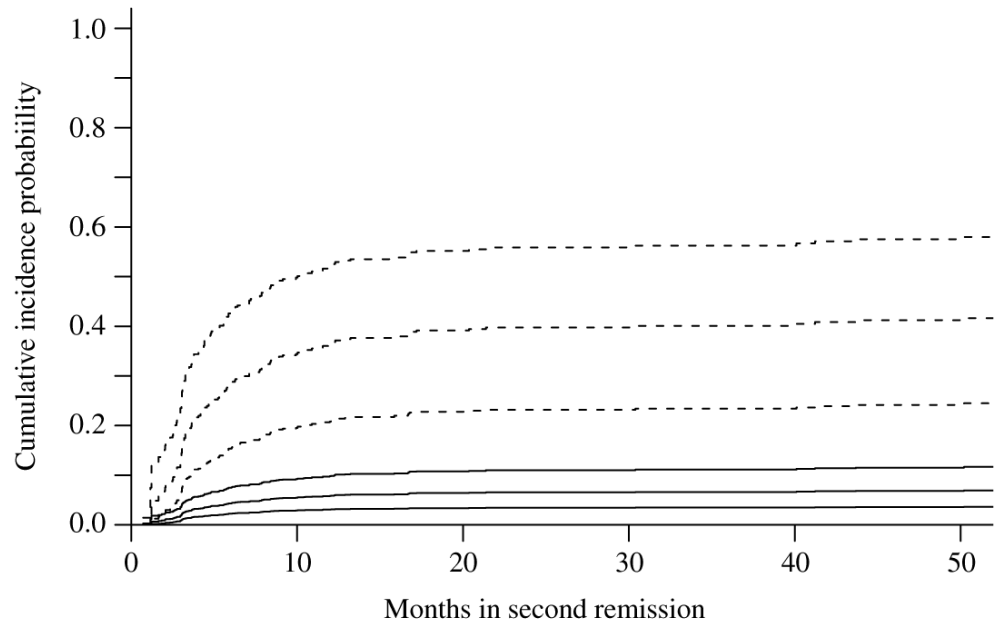

Figure 2.

Predicted cumulative incidences of TRM for chemotherapy (solid) and BMT (dashes) based on a boy without T-cell phenotype and diagnosed after 1984. 


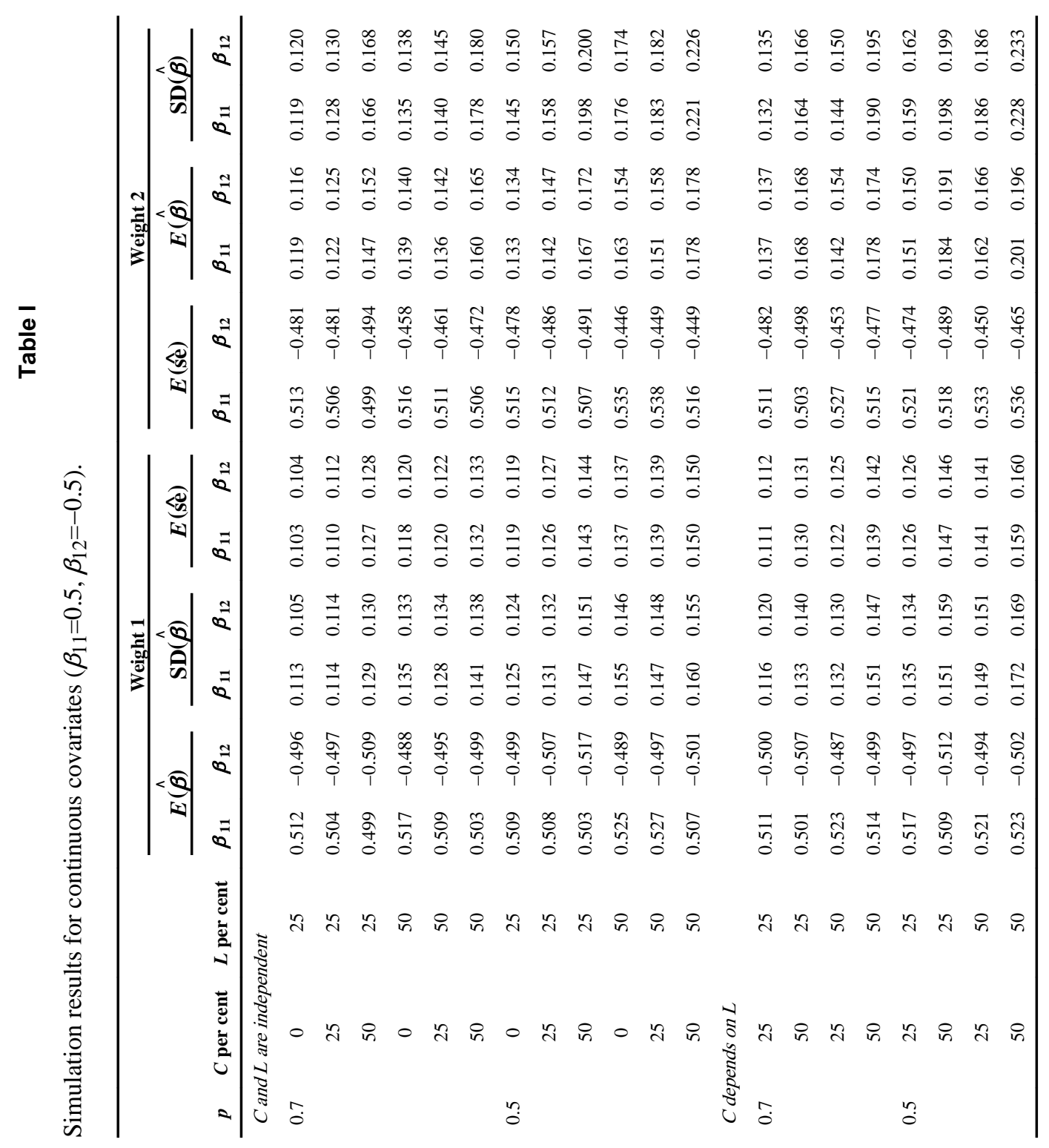




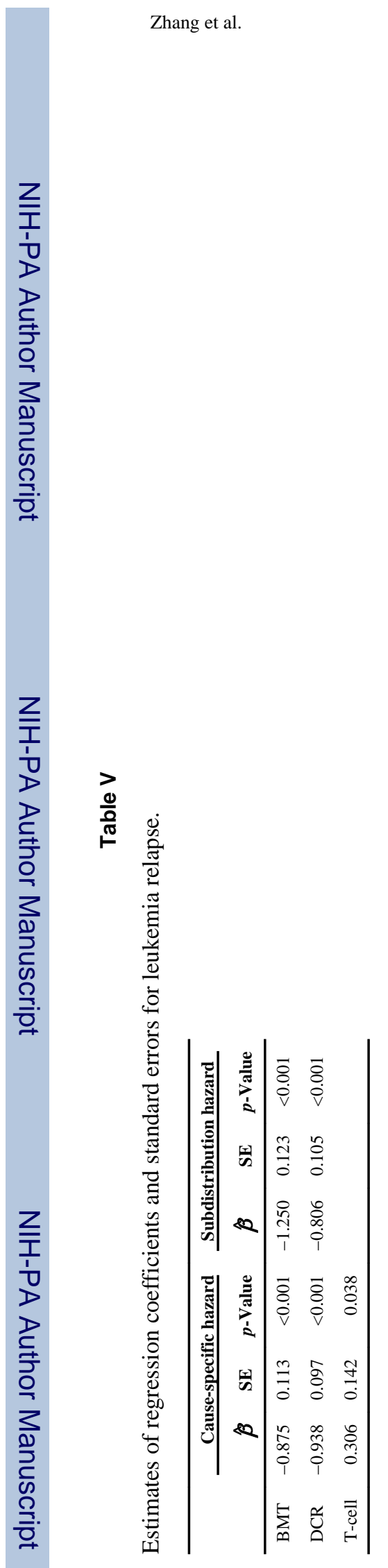

Stat Med. Author manuscript; available in PMC 2012 July 31. 


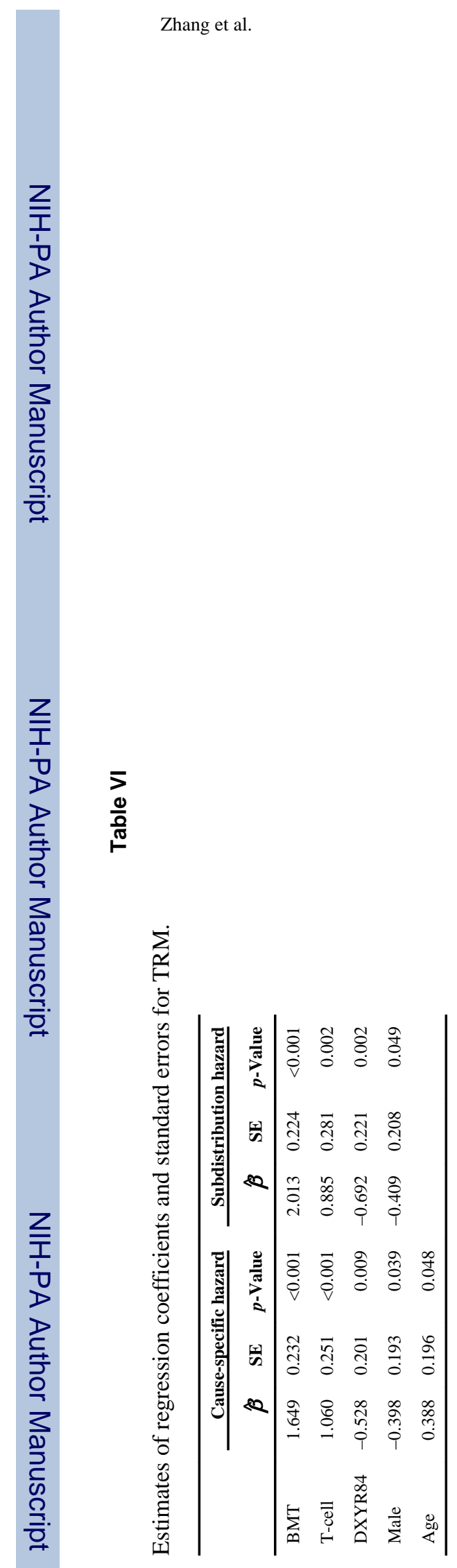

Page 26

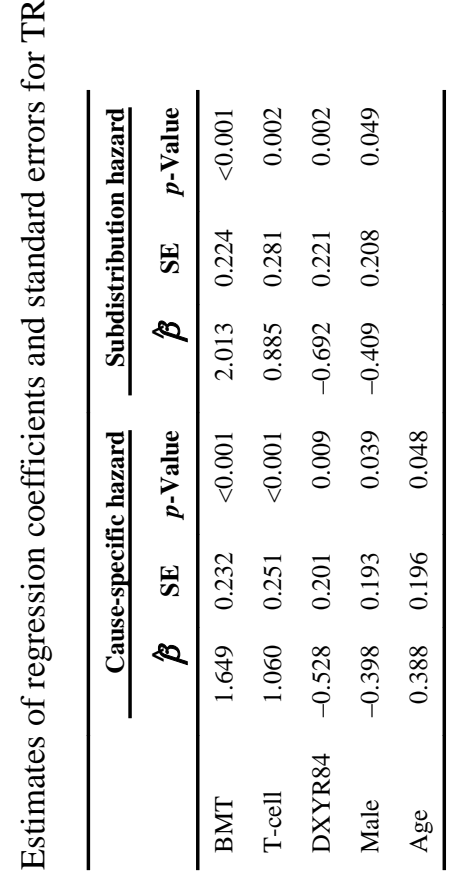

Stat Med. Author manuscript; available in PMC 2012 July 31. 